\title{
Reachability on prefix-recognizable graphs
}

\author{
Stefan Göller \\ Institute for Computer Science \\ University of Leipzig, Germany
}

\begin{abstract}
We prove that on prefix-recognizable graphs reachability is complete for deterministic exponential time matching the complexity of alternating reachability.
\end{abstract}

Key words: Reachability, Prefix-recognizable graphs, Computational complexity, Alternation

\section{Introduction}

The reachability problem asks, given some graph $G$ and two vertices $u$ and $v$ of $G$, if there exists a path from $u$ to $v$ in $G$. The alternating reachability problem asks, given two vertices $u$ and $v$ of some game graph, i.e. a graph whose vertices are partitioned into those for player Eve and player Adam, if Eve has a winning strategy in the reachability game from $u$ to $v$. On finite graphs it is well known that the reachability problem is complete for NL, whereas for the alternating reachability problem the complexity jumps to $\mathrm{P}$-completeness.

It is natural to ask what the complexity status of the (alternating) reachability problem is when the input graph is potentially infinite but finitely presented. On pushdown graphs reachability can be solved in deterministic polynomial time [2] and a matching lower bound can easily be shown via a reduction from the nonemptiness problem of context-free grammars. Alternating reachability on pushdown graphs on the other hand is EXP-complete [14]. On one-counter graphs, i.e. transition graphs of pushdown automata over a singleton stack alphabet, reachability is NL-complete, whereupon recent results $[9,13]$ imply that alternating reachability is PSPACE-complete. Reachability on order- $n$ pushdown graphs (which generalize pushdown graphs) is (n-1)-EXP-complete [7],

Email address: goeller@informatik.uni-leipzig.de (Stefan Göller). 
whereas alternating reachability is $n$-EXP-complete [8,4]. Summarizing, for the above mentioned established classes of infinite graphs, there is an exponential complexity jump from reachability to alternating reachability. A comparable phenomenon can be observed on regular ground tree rewriting graphs: Reachability is decidable but already deciding whether all paths starting in some source vertex eventually reach some vertex of a regular set of vertices is undecidable [12]. Even worse, on the more general class of automatic graphs, where reachability is $\Sigma_{1}^{0}$-complete [1], alternating reachability is at least $\Pi_{1}^{1}$-hard following from a recent result [10], where it is shown inter alia that deciding if an automatic successor tree has an infinite path is $\Sigma_{1}^{1}$-complete.

Prefix-recognizable graphs, introduced by Caucal [5], form a natural class of infinite graphs with numerous characterizations and a decidable monadic second order theory; [11] gives a nice overview. For instance, they can be characterized as the transition graphs of pushdown automata with $\varepsilon$-transitions, thus generalizing (order-1) pushdown graphs. We prove that already the reachability problem on prefix-recognizable graphs is EXP-hard. Since solving parity games on prefix-recognizable is in EXP [3], it immediately follows that alternating reachability is in EXP too. Thus surprisingly, both reachability and alternating reachability on prefix-recognizable graphs are EXP-complete. So alternation on prefix-recognizable graphs comes for free.

\section{Preliminaries}

For a finite alphabet $\Sigma$ and languages $U, V, W \subseteq \Sigma^{*}$, let $(U \times V) W$ denote the binary relation $\{(u w, v w) \mid u \in U, v \in V, w \in W\}$. For simplicity, a prefix-recognizable graph is a binary relation $G \subseteq \Sigma^{*} \times \Sigma^{*}$ such that for some $k \geq 1$ there exist regular languages $U_{1}, V_{1}, W_{1}, \ldots, U_{k}, V_{k}, W_{k}$ over $\Sigma$ such that $G=\cup_{i=1}^{k}\left(U_{i} \times V_{i}\right) W_{i}$. We assume that $G$ is represented by a set of triples of regular expressions $\left(\alpha_{i}, \beta_{i}, \gamma_{i}\right)_{1 \leq i \leq k}$ where $L\left(\alpha_{i}\right)=U_{i}, L\left(\beta_{i}\right)=V_{i}, L\left(\gamma_{i}\right)=W_{i}$. The size of $G$ is defined as $\sum_{i=1}^{k}\left|\alpha_{i}\right|+\left|\beta_{i}\right|+\left|\gamma_{i}\right|$.

The reachability problem (on prefix-recognizable graphs) is stated as follows:

INPUT: A prefix-recognizable graph $G$ and $u, v \in \Sigma^{*}$. QUESTION: $(u, v) \in G^{+}$?

For proving an EXP lower bound for reachability on prefix-recognizable graphs we need to introduce alternating Turing machines. An alternating Turing machine $(A T M)$ is a tuple $\mathcal{M}=\left(Q, \Gamma, \Lambda, q_{0}, \delta, \square\right)$ where (i) $Q=Q_{\forall} \uplus Q_{\exists}$ is a finite set of states, which is partitioned into universal states $Q_{\forall}$ and existential states $Q_{\exists}$, (ii) $\Gamma$ is a finite tape alphabet, (iii) $\Lambda \subseteq \Gamma$ is the input alphabet, (iv) $q_{0} \in Q$ is the initial state, (v) $\square \in \Gamma \backslash \Lambda$ is the blank symbol, and (vi) the mapping $\delta: Q \times \Gamma \rightarrow$ (Moves $\times$ Moves) $\cup\{\perp\}$ with Moves $=Q \times \Gamma \times\{\leftarrow, \rightarrow\}$ 
assigns to every pair $(q, a) \in Q \times \Gamma$ either a pair of moves or $\perp$. Hence, we assume that every configuration of an ATM either has two successor configurations or none. An acceptance tree $T$ of a configuration $u$ of $\mathcal{M}$ that is in current state $q \in Q$ and scans a symbol $a \in \Gamma$ is a finite tree with root $u$ such that exactly one of the following three conditions holds:

(a) $q \in Q_{\forall}, \delta(q, a)=\perp$ and $T$ is a singleton (with root $u$ ).

(b) $q \in Q_{\forall}, \delta(q, a) \neq \perp$, i.e. $u$ has two successor configurations $u_{1}$ and $u_{2}$, and $T$ consists of the root $u$ together with two subtrees $T_{1}$ and $T_{2}$, where $T_{1}$ (resp. $T_{2}$ ) is some acceptance tree of $u_{1}$ (resp. $u_{2}$ ).

(c) $q \in Q_{\exists}, \delta(q, a) \neq \perp$ and $T$ consists of the root $u$ together with one subtree $T^{\prime}$, where $T^{\prime}$ is an acceptance tree of one of the two successor configurations of $u$.

The height of an acceptance tree $T$ is the largest distance from some leaf of $T$ to the root of $T$. We call a configuration of $\mathcal{M}$ accepting if it has an acceptance tree. The language of $\mathcal{M}$ is defined as $L(\mathcal{M})=\left\{w \in \Lambda^{*} \mid\right.$ configuration $q_{0} w$ is accepting $\}$. We denote by EXP the class of problems that are decidable by some $2^{p(n)}$ time bounded deterministic Turing machine for some polynomial $p$.

\section{EXP-hardness of reachability on prefix-recognizable graphs}

We proceed with the EXP lower bound for reachability on prefix-recognizable graphs. For this, fix some $p(n)$-space bounded ATM $\mathcal{M}=\left(Q, \Lambda, \Gamma, q_{0}, \delta, \square\right)$, for some polynomial $p$, with an EXP-hard membership problem, which exists by [6]. Moreover, let $w \in \Lambda^{*}$ be an input of length $n$ and let $N=p(n)$. Configurations of $\mathcal{M}$ will be described by the regular language

$$
U=\bigcup_{0 \leq i<N} \Gamma^{i} Q \Gamma^{N-i}
$$

In the following, configurations of $\mathcal{M}$ will be confused with members of $U$ and vice versa. We will construct a polynomial time computable prefix-recognizable graph $G$ such that

$$
w \in L(\mathcal{M}) \quad \Leftrightarrow \quad\left(q_{0} w \square^{N-n}, \varepsilon\right) \in G^{+} .
$$

We may assume w.l.o.g. that $\mathcal{M}$ never attempts to move left (right) scanning the left-most (right-most) tape cell. Let Moves denote the moves that occur as components in $\delta(Q, \Gamma) \backslash\{\perp\}$. The alphabet of $G$ will be

$$
\Sigma=Q \cup \Gamma \cup\{l, r, e\} \cup\left\{\sigma_{i, j} \mid \sigma \in \text { Moves and } i, j \in[0, N+1]\right\} \text {. }
$$


Before defining $G$, let us introduce a relation $\longrightarrow \subseteq \Sigma^{*} \times \Sigma^{*}$ that describes a single step of a depth-first left-to-right traversal of acceptance trees of $\mathcal{M}$. For this, let $u$ range over $U$ being in state $q \in Q$, scanning a symbol $a \in \Gamma$, and where $v \in \Sigma^{*}$ is arbitrary,

$$
\begin{aligned}
& u v \quad \longrightarrow \quad v \quad \text { if } q \in Q_{\forall} \text { and } \delta(q, a)=\perp, \\
& x u v \longrightarrow v \quad \text { if } x \in\{e, r\} \text {, } \\
& u v \quad \longrightarrow \quad u^{\prime} l u v \quad \text { if } q \in Q_{\forall}, \delta(q, a)=(\sigma, \tau) \in \text { Moves }^{2} \text {, and } u^{\prime} \text { is } \\
& \text { the } \sigma \text {-successor configuration of } u \text {, } \\
& u v \quad \longrightarrow \quad u^{\prime} \text { euv } \quad \text { if } q \in Q_{\exists}, \delta(q, a)=(\sigma, \tau) \in \text { Moves }^{2} \text { and } u^{\prime} \text { is } \\
& \text { the } \sigma \text { - or } \tau \text {-successor configuration of } u \text {, } \\
& \text { luv } \longrightarrow u^{\prime} r u v \quad \text { if } q \in Q_{\forall}, \delta(q, a)=(\sigma, \tau) \in \text { Moves }^{2} \text {, and } u^{\prime} \text { is } \\
& \text { the } \tau \text {-successor configuration of } u \text {. }
\end{aligned}
$$

Next, we prove that the relation $\longrightarrow$ is as required.

Lemma 1 For every $u \in U$ the following three statements are equivalent:

(i) $u \longrightarrow^{+} \varepsilon$,

(ii) $u v \longrightarrow^{+} v$ for every $v \in \Sigma^{*}$,

(iii) $u$ is accepting.

\section{PROOF.}

(ii) $\Rightarrow$ (i): Trivial.

(i) $\Rightarrow$ (iii): We prove $u \longrightarrow^{k} \varepsilon$ implies that $u$ has an acceptance tree by induction on $k \geq 1$. The case $k=1$ is clear since we can only apply (1) and $u$ has an acceptance tree by (a). Now assume $u \longrightarrow^{k+1} \varepsilon$. Assume $u$ is universal and let $u^{\prime}, u^{\prime \prime} \in U$ be the two successor configurations of $u$. It follows that $u \longrightarrow{ }^{k+1} \varepsilon$ is decomposed as

$$
u \stackrel{(3)}{\longrightarrow} u^{\prime} l u \longrightarrow{ }^{k_{1}} l u \stackrel{(5)}{\longrightarrow} u^{\prime \prime} r u \longrightarrow^{k_{2}} r u \stackrel{(2)}{\longrightarrow} \varepsilon
$$

where $k_{1}+k_{2}<k$. The latter is due to the observation that every occurrence of the letter $l$ (resp. $r$ ) in a word $z \in \Sigma^{*}$ can only be rewritten by $\longrightarrow$ if $l$ (resp. $r$ ) is the first letter of $z$. Moreover it follows by the definition of $\longrightarrow$ that $u^{\prime} \longrightarrow^{k_{1}} \varepsilon$ and $u^{\prime \prime} \longrightarrow{ }^{k_{2}} \varepsilon$. Hence, by induction hypothesis both for $u^{\prime}$ and $u^{\prime \prime}$ there exists an acceptance tree. Thus, by (b), there exists an acceptance tree for $u$ too. The case when $u$ is existential can be proven analogously.

(iii) $\Rightarrow$ (ii): Assume $u$ is accepting. We prove $u v \longrightarrow^{+} v$ for all $v \in \Sigma^{*}$ by induction on the smallest height $h$ of some acceptance tree of $u$. Assume $h=0$, hence $u$ is in some state $q \in Q_{\forall}$ scanning some symbol $a \in \Gamma$ and $\delta(q, a)=\perp$ by (a). Thus, by applying (1), we obtain $u v \longrightarrow v$ for all $v \in \Sigma^{*}$. 

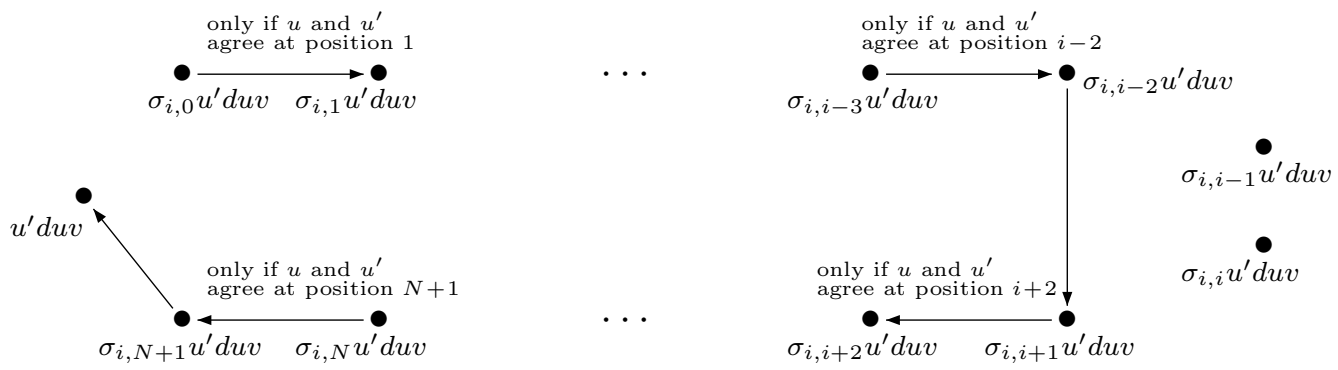

Fig. 1. The graph $G_{=}$

Now let $h>0$. In case $u$ is existential, there exists a successor configuration $u^{\prime}$ of $u$ for which the smallest height of some acceptance tree for $u^{\prime}$ is strictly less than $h$. Hence, by induction hypothesis, we have $u^{\prime} v^{\prime} \longrightarrow{ }^{+} v^{\prime}$ for all $v^{\prime} \in \Sigma^{*}$. By additionally applying (2) and (4) we obtain for every $v \in \Sigma^{*}$

$$
u v \stackrel{(4)}{\longrightarrow} u^{\prime} \text { euv } \longrightarrow+\stackrel{(2)}{\longrightarrow} v .
$$

Hence $u v \longrightarrow^{+} v$ for all $v \in \Sigma^{*}$. The case when $u$ is universal can be proven analogously.

Our prefix-recognizable graph $G$ will be the union of several prefix-recognizable graphs we define below.

The prefix-recognizable graph $G_{1}$ corresponds to (1):

$$
G_{1}=\bigcup_{\substack{0 \leq i<N \\ q \in Q_{\forall}, a \in \Gamma: \delta(q, a)=\perp}}\left(\Gamma^{i} q a \Gamma^{N-i-1} \times\{\varepsilon\}\right) \Sigma^{*}
$$

Similarly, the prefix-recognizable graph $G_{2}$ corresponds to (2):

$$
G_{2}=(\{e, r\} U \times\{\varepsilon\}) \Sigma^{*}
$$

Before defining prefix-recognizable graphs that correspond to (3), (4), and (5) respectively, we need an auxiliary graph $G_{=}$that is often used later and has the following property: For each $\sigma \in$ Moves and each $u, u^{\prime} \in U$ we have $\left(\sigma_{i, 0} u^{\prime} d u v, u^{\prime} d u v\right) \in G_{=}^{+}$only if $u^{\prime}$ agrees with $u$ on all positions besides $\{i-$ $1, i, i+1\}$, where $d \in\{l, r, e\}$ and $v \in \Sigma^{*}$. Figure 1 shows how this can be achieved. Formally, we define 


$$
\begin{gathered}
G==\bigcup_{\substack{1<i \leq N, \sigma \in \text { Moves }}}\left(\left\{\sigma_{i, i-2}\right\} \times\left\{\sigma_{i, i+1}\right\}\right) \Sigma^{*} \cup\left(\sigma_{i, N+1} \times\{\varepsilon\}\right) \Sigma^{*} \cup \\
\bigcup_{0 \leq j<i-2}^{\substack{\text { or } i+1 \leq j \leq N \\
c \in \Gamma}}\left(\left\{\sigma_{i, j}\right\} \times\left\{\sigma_{i, j+1}\right\}\right) \Sigma^{j} c \Sigma^{N+1} c \Sigma^{*} .
\end{gathered}
$$

Let us turn to a graph $G_{3}$ that corresponds to (3). We make a case distinction. In case the move $\sigma$ is from $Q \times \Gamma \times\{\longleftarrow\}$, we realize (3) by

$$
G_{3}^{\leftarrow}=\bigcup_{1<i \leq N} \bigcup_{\substack { c \in \Gamma \\
\begin{subarray}{c}{q \in Q \forall, a \in \Gamma \\
\delta(q, a)=(\sigma, \tau): \\
\sigma=\left(q^{\prime}, b, \leftarrow\right){ c \in \Gamma \\
\begin{subarray} { c } { q \in Q \forall , a \in \Gamma \\
\delta ( q , a ) = ( \sigma , \tau ) : \\
\sigma = ( q ^ { \prime } , b , \leftarrow ) } }\end{subarray}}\left(\{\varepsilon\} \times \sigma_{i, 0} \Gamma^{i-2} q^{\prime} c b \Gamma^{N-i} l\right) \Gamma^{i-2} c q a \Gamma^{N-i} \Sigma^{*} .
$$

In case $\sigma$ is from $Q \times \Gamma \times\{\rightarrow\}$, we realize (3) by

$$
G_{3}^{\rightarrow}=\bigcup_{1<i \leq N} \bigcup_{\substack { c \in \Gamma \\
\begin{subarray}{c}{q \in Q, a \in \Gamma \\
\delta(q, a)=(\sigma, \tau): \\
\sigma=\left(q^{\prime}, b, \rightarrow\right){ c \in \Gamma \\
\begin{subarray} { c } { q \in Q , a \in \Gamma \\
\delta ( q , a ) = ( \sigma , \tau ) : \\
\sigma = ( q ^ { \prime } , b , \rightarrow ) } }\end{subarray}}\left(\{\varepsilon\} \times \sigma_{i, 0} \Gamma^{i-2} b q^{\prime} c \Gamma^{N-i} l\right) \Gamma^{i-2} q a c \Gamma^{N-i} \Sigma^{*}
$$

Define $G_{3}=G_{3}^{\leftarrow} \cup G_{3}^{\rightarrow}$. Note that both $G_{3}^{\rightarrow}$ and $G_{3}^{\leftarrow}$ allow to get from vertices $u v$ to vertices $\sigma_{i, 0} u^{\prime} l u v$ where $u^{\prime}$ is not necessarily the $\sigma$-sucessor of $u$. But thanks to $G_{=}$it is guaranteed that from $\sigma_{i, 0} u^{\prime} l u v$ the vertex $u^{\prime} l u v$ can only be reached if $u^{\prime}$ is indeed the $\sigma$-successor of $u$.

Analogously to (3), the graph $G_{4}$ that corresponds to (4) is the union of

$$
\bigcup_{1<i \leq N} \bigcup_{c \in \Gamma} \bigcup_{\substack{q \in Q_{\exists}, a \in \Gamma \\ \delta(q, a)=\left(\tau, \tau^{\prime}\right): \\ \sigma=\left(q^{\prime}, b, \leftarrow\right) \in\left\{\tau, \tau^{\prime}\right\}}}\left(\{\varepsilon\} \times \sigma_{i, 0} \Gamma^{i-2} q^{\prime} c b \Gamma^{N-i} e\right) \Gamma^{i-2} c q a \Gamma^{N-i} \Sigma^{*}
$$

and

$$
\bigcup_{1<i \leq N} \bigcup_{c \in \Gamma} \bigcup_{\substack{q \in Q \\ \delta(q, a)=\left(\tau, \tau^{\prime}\right): \\ \sigma=\left(q^{\prime}, b, \rightarrow \in\left\{\tau, \tau^{\prime}\right\}\right.}}\left(\{\varepsilon\} \times \sigma_{i, 0} \Gamma^{i-2} b q^{\prime} c \Gamma^{N-i} e\right) \Gamma^{i-2} q a c \Gamma^{N-i} \Sigma^{*}
$$

Finally, as for (3) and (4), the graph $G_{5}$ that realizes (5) is the union of

and

$$
\bigcup_{1<i \leq N} \bigcup_{c \in \Gamma} \bigcup_{\substack{q \in Q \forall, a \in \Gamma \\ \delta(q, a)=(\sigma, \tau): \\ \tau=\left(q^{\prime}, b, \leftarrow\right)}}\left(\{l\} \times \tau_{i, 0} \Gamma^{i-2} q^{\prime} c b \Gamma^{N-i} r\right) \Gamma^{i-2} c q a \Gamma^{N-i} \Sigma^{*}
$$

$$
\bigcup_{1<i \leq N} \bigcup_{\substack{c \in \Gamma \\ c \in c}} \bigcup_{\substack{q \in Q, a \in \Gamma \\ \delta(q, a)=(\sigma, \tau): \\ \tau=\left(q^{\prime}, b, \rightarrow\right)}}\left(\{l\} \times \tau_{i, 0} \Gamma^{i-2} b q^{\prime} c \Gamma^{N-i} r\right) \Gamma^{i-2} q a c \Gamma^{N-i} \Sigma^{*}
$$


Define $G=G_{1} \cup G_{2} \cup G_{3} \cup G_{4} \cup G_{5} \cup G_{=}$. It is easy to verify that $G$ is computable in time polynomial in $n=|w|$. The following fact is not hard to check.

Fact 2 Let $A=(U \cup\{\varepsilon\})(\{l, r, e\} U)^{*}$. Then

$$
\longrightarrow^{+} \cap A \times A=G^{+} \cap A \times A \text {. }
$$

The following equivalences finish our EXP lower bound proof:

$$
\begin{aligned}
& w \in L(\mathcal{M}) \quad \Longleftrightarrow \quad q_{0} w \square^{N-n} \in U \text { is accepting } \\
& \stackrel{\text { Lemma }}{\Longleftrightarrow} \quad \forall v \in \Sigma^{*}: q_{0} w \square^{N-n} v \longrightarrow^{+} v \\
& \stackrel{\text { Lemma }}{\Longleftrightarrow} \quad q_{0} w \square^{N-n} \longrightarrow{ }^{+} \varepsilon \\
& \stackrel{\text { Fact } 2}{\Longleftrightarrow} \quad\left(q_{0} w \square^{N-n}, \varepsilon\right) \in G^{+} \text {. }
\end{aligned}
$$

Since solving parity games on prefix-recognizable graphs is in EXP [3], we get the following theorem.

Theorem 3 The reachability problem on prefix-recognizable graphs is EXPcomplete.

It is a natural question to ask what the complexity of the reachability problem is on prefix rewrite graphs, where transitions are given as

$$
\bigcup_{1 \leq i \leq k}\left(U_{i} \times V_{i}\right) \Sigma^{*} \quad \text { for regular languages } U_{1}, V_{1}, \ldots, U_{k}, V_{k} \text {. }
$$

Note that in our lower bound proof only $G_{=}$was not of the form $(*)$. The latter is inevitable, since there is an easy reduction from the reachability problem on prefix rewrite graphs to the reachability problem on pushdown graphs: Transitions of the kind $\left(U_{i} \times V_{i}\right) \Sigma^{*}$ are simulated by a sequence of transitions of two nondeterministic finite automata that simulate $U_{i}$ (resp. $V_{i}$ ) by popping (resp. pushing) symbols from (resp. on) the stack. Thus, reachability on prefix rewrite graphs is $\mathrm{P}$-complete.

Acknowledgments The author would like to thank Markus Lohrey and Christian Mathissen for discussion and proofreading.

\section{References}

[1] Achim Blumensath and Erich Grädel. Finite presentations of infinite structures: Automata and interpretations. Theory of Comput. Syst., 37(6):641-674, 2004. 
[2] Ahmed Bouajjani, Javier Esparza, and Oded Maler. Reachability analysis of pushdown automata: Application to model-checking. In CONCUR, number 1243 in Lecture Notes in Computer Science, pages 135-150. Springer, 1997.

[3] Thierry Cachat. Uniform solution of parity games on prefix-recognizable graphs. Electr. Notes Theor. Comput. Sci., 68(6), 2002.

[4] Thierry Cachat and Igor Walukiewicz. The complexity of games on higher order pushdown automata. CoRR, abs/0705.0262, 2007.

[5] Didier Caucal. On infinite transition graphs having a decidable monadic theory. Theoretical Computer Science, 290(1):79-115, 2002.

[6] Ashok Chandra, Dexter Kozen, and Larry Stockmeyer. Alternation. Journal of the Association for Computing Machinery, 28(1):114-133, 1981.

[7] Joost Engelfriet. Iterated pushdown automata and complexity classes. In STOC, pages 365-373. ACM, 1983.

[8] Matthew Hague and Luke Ong. Symbolic backwards-reachability analysis for higher-order pushdown systems. In FoSSaCS, volume 4423 of Lecture Notes in Computer Science, pages 213-227. Springer, 2007.

[9] Petr Jancar and Zdenek Sawa. A note on emptiness for alternating finite automata with a one-letter alphabet. Inf. Process. Lett., 104(5):164-167, 2007.

[10] Dietrich Kuske and Markus Lohrey. Hamiltonicity of automatic graphs. To appear in Proceedings of IFIP-TCS, 2008.

[11] Martin Leucker. Prefix-recognizable graphs and monadic logic. In Automata, Logics, and Infinite Games, Lecture Notes in Computer Science, pages 263-284. Springer, 2001.

[12] Christof Löding. Reachability problems on regular ground tree rewriting graphs. Theory Comput. Syst., 39(2):347-383, 2006.

[13] Olivier Serre. Parity games played on transition graphs of one-counter processes. In FoSSaCS, volume 3921 of Lecture Notes in Computer Science, pages 337-351. Springer, 2006.

[14] Dejvuth Suwimonteerabuth, Stefan Schwoon, and Javier Esparza. Efficient algorithms for alternating pushdown systems with an application to the computation of certificate chains. In ATVA, volume 4218 of Lecture Notes in Computer Science, pages 141-153. Springer, 2006. 